\title{
Concurrent analysis of human equilibrative nucleoside transporter 1 and ribonucleotide reductase subunit 1 expression increases predictive value for prognosis in cholangiocarcinoma patients treated with adjuvant gemcitabine-based chemotherapy
}

\author{
H Sasaki ${ }^{*}{ }^{1}$, Y Murakami ${ }^{1}$, K Uemura ${ }^{1}$, T Sudo $^{1}$, Y Hashimoto $^{1}, \mathrm{~N}$ Kondo ${ }^{1}$ and T Sueda ${ }^{1}$ \\ ${ }^{1}$ Department of Surgery, Institute of Biomedical and Health Sciences, Hiroshima University, Hiroshima 734-8551, Japan
}

\begin{abstract}
Background: The aim of this study was to investigate the predictive and prognostic values of intratumoural human equilibrative nucleoside transporter 1 (hENT1) and ribonucleotide reductase subunit 1 (RRM1) expression in advanced cholangiocarcinoma patients treated with adjuvant gemcitabine-based chemotherapy (AGC).
\end{abstract}

Methods: Intratumoural hENT1 and RRM1 expression levels were investigated immunohistochemically in 127 patients with advanced cholangiocarcinoma who underwent surgical resection ( 68 with AGC and 59 without AGC). The impacts of hENT1 and RRM1 expression on survival were evaluated.

Results: High intratumoural hENT1 and RRM1 expression levels were observed in 86 (68\%) and 67 (53\%) patients, respectively. In a multivariate analysis of 68 patients who received AGC, high hENT1 $(P=0.044)$ and low RRM1 expression $(P=0.009)$ were independently associated with prolonged disease-free survival (DFS), whereas low RRM1 expression $(P=0.024)$ was independently associated with prolonged overall survival (OS). Moreover, concurrent high hENT1 and low RRM1 expression was a powerful independent predictor of prolonged DFS $(P<0.001)$ and OS $(P=0.001)$ when the combined classification of hENT1 and RRM1 was introduced.

Conclusions: Concurrent analysis of hENT1 and RRM1 expression may increase the predictive value of these biomarkers for survival of advanced cholangiocarcinoma patients treated with AGC.

Cholangiocarcinoma, including intra- and extrahepatic cholangiocarcinoma, is a relatively uncommon disease in the United States, accounting for 4410 deaths in 2012 (Siegel et al, 2013). However, this disease is the sixth leading cause of cancer-related deaths in Japan, with $>18000$ deaths reported in 2012 (National Cancer Center, Japan, 2014). Although surgical resection is the only curative treatment for cholangiocarcinoma, the 5-year overall survival (OS) rates of patients with resected cholangiocarcinoma are still 18-40\%, even in high-volume centers (DeOliveira et al, 2007; Murakami et al, 2011; Nagino et $a l, 2013)$. Therefore, several peri-operative therapeutic modalities, including adjuvant chemotherapy, have recently been proposed in order to improve the prognosis of patients with cholangiocarcinoma. 
Since 2002, post-operative adjuvant gemcitabine-based chemotherapy (AGC) has been administered to patients with advanced cholangiocarcinoma (International Union Against Cancer (UICC) stages II-IV) in our institution, and we have previously reported a survival benefit associated with this therapy (Murakami et al, 2009, 2011, 2012). However, the efficacy of AGC varies among individuals, and the resulting survival rates are still unsatisfactory. To maximise the therapeutic benefit of adjuvant chemotherapy, identification of biomarkers that have predictive and prognostic value is important. Several clinical studies have revealed the predictive significance of intratumoural human equilibrative nucleoside transporter 1 (hENT1) for survival in pancreatic cancer patients treated with gemcitabine (Spratlin et al, 2004; Giovannetti et al, 2006; Farrell et al, 2009; Maréchal et al, 2012; Wei et al, 2013; Greenhalf et al, 2014). Moreover, our recent report demonstrated that hENT1 also predicts the survival of cholangiocarcinoma patients treated with AGC (Kobayashi et al, 2012). Thus, hENT1 has been recognised as a relevant predictive biomarker for response to gemcitabine.

In addition, researchers have recently also become interested in identifying other candidates for predictive biomarkers related to gemcitabine sensitivity. In particular, the expression of ribonucleotide reductase subunit M1 (RRM1), which is involved in the production of deoxyribonucleotides for DNA synthesis, has been reported to be associated with gemcitabine resistance in several cancers (Ohtaka et al, 2008; Akita et al, 2009; Jordheim et al, 2011; Gong et al, 2012). In addition, we have recently demonstrated that combined analysis of hENT1 and RRM1 expression was a more powerful predictor than analysis of either target alone in pancreatic cancer (Nakagawa et al, 2013). However, very few reports have revealed the predictive significance of RRM1 expression for gemcitabine resistance in cholangiocarcinoma. In addition, the predictive value of combined analysis of hENT1 and RRM1 expression in cholangiocarcinoma is still unclear. The aim of this study was to investigate the predictive and prognostic values of intratumoural hENT1 and RRM1 expression in advanced cholangiocarcinoma patients treated with AGC after surgical resection.

\section{MATERIALS AND METHODS}

Study design. Patients with advanced cholangiocarcinoma (UICC stages II, IIA, IIB, III, IIIA, IIIB, IV, IVA, and IVB) who underwent surgical resection with curative intent (R0 or R1 resection) at the Department of Surgery, Hiroshima University Hospital, Hiroshima, Japan between April 1989 and August 2012 were enrolled in this study. All patients had a confirmed pathological diagnosis. Patients who experienced post-operative mortality were excluded from this study. Formalin-fixed, paraffin-embedded tumour tissues from the resected specimens were collected from eligible patients, and immunohistochemical staining for detection of intratumoural hENT1 and RRM1 was performed. The influences of clinicopathological factors and hENT1 and RRM1 expression on survival were evaluated by univariate and multivariate analyses. Written informed consent was obtained from all patients for surgical treatment and pathological examinations, as required by institutional guidelines.

Surgical procedures and pathological assessment. Most patients with intrahepatic or perihilar cholangiocarcinoma underwent major hepatectomy, and all surgical procedures for perihilar cholangiocarcinoma included caudate lobectomy. Patients with distal cholangiocarcinoma usually underwent pancreatoduodenectomy with or without pylorus preservation. Dissection of the regional lymph nodes was performed for all patients. All resected specimens were examined histologically by specialised pathologists; each tumour was classified as well-differentiated, moderately differentiated, or poorly differentiated adenocarcinoma according to the predominant histology. Residual tumour ( $\mathrm{R}$ factor) was considered R1 if histological infiltrating carcinoma was present at the proximal or distal bile duct transaction line, the hepatic transaction line, or the dissected peripancreatic soft-tissue margins. All patients with R2 resections were excluded from this study. Tumour stage, lymph node metastasis, and final stage were classified based on the 7th edition of the UICC tumour-nodemetastasis (TMN) classification (Sobin et al, 2010).

Adjuvant gemcitabine-based chemotherapy. The AGC regimen used in this study, which included two treatment options, has been reported previously (Murakami et al, 2009, 2011, 2012). First, intravenous chemotherapy consisted of gemcitabine $700 \mathrm{mg} \mathrm{m}^{-2}$ administered biweekly. Second, intravenous and oral chemotherapy consisted of intravenous gemcitabine $700 \mathrm{mg} \mathrm{m}^{-2}$ on day 1 and oral S-1 $50 \mathrm{mg} \mathrm{m}^{-2}$ for 7 consecutive days. These regimens were repeated every 14 days for 10 cycles. None of the patients received radiation therapy during this study period. Patients who had to switch to other chemotherapies before the 10 cycles were completed because of recurrent disease were considered to have received AGC in our group classification. Patients who received gemcitabine-based chemotherapy because of recurrent disease after completion of AGC were also included in this group.

Immunohistochemistry for hENT1 and RRM1. Polyclonal rabbit antibodies against human hENT1 (Abnova Co., Taipei, Taiwan) and against human RRM1 (Abcam, Cambridge, UK) were used to evaluate hENT1 and RRM1 expression, respectively. Following antigen retrieval by autoclaving $\left(100^{\circ} \mathrm{C}\right.$ for $10 \mathrm{~min}$ in Dako Target Retrieval Solution High pH $1 \times$ for hENT1 or $121^{\circ} \mathrm{C}, 10 \mathrm{~min}$ in $0.01 \mathrm{M}$ citrate buffer for RRM1), sections were immersed in methanol containing 3\% hydrogen peroxide for $15 \mathrm{~min}$, incubated in protein blocking solution (Dako, Carpinteria, CA, USA) for $10 \mathrm{~min}$, and incubated with anti-hENT1 antibodies $(1: 200$ dilution) overnight at $4{ }^{\circ} \mathrm{C}$ or anti-RRM1 antibodies (1:150 dilution) for $60 \mathrm{~min}$ at room temperature. Samples were then incubated in labelled streptavidin-biotin polymer (Envision Plus, Dako, Hamburg, Germany) at room temperature for $60 \mathrm{~min}$ as a secondary antibody and immersed for $10 \mathrm{~min}$ in $0.01 \% 3,3$ diaminobenzidine solution in $50 \mathrm{~mm}$ Tris- $\mathrm{HCl}$ buffer with $10 \mathrm{~mm}$ hydrogen peroxide as a substrate. Sections were counterstained with Mayer's haematoxylin, dehydrated, and mounted. Negative controls were provided by omitting the primary antibodies.

Two observers (HS and NK), blinded to clinical characteristics and outcomes, assessed and scored the expression of hENT1 and RRM1. In cases of disagreement, consensus was reached by joint review. Because hENT1 is strongly expressed in cell membranes of lymphocytes (Spratlin et al; 2004; Farrell et al, 2009), and RRM1 is strongly expressed in plasma and stromal cells (Ohtaka et al, 2008; Akita et al, 2009), these were used as internal positive controls. The intensities of hENT1 and RRM1 staining were scored as follows: grade 0 , not stained; grade 1 , weakly stained compared with the internal positive control; grade 2, stained as strongly as the internal positive control; and grade 3, strongly stained compared with the internal positive control. For evaluation of intratumoural hENT1 and RRM1 expression, if grade 2 or 3 staining was observed in $>50 \%$ of tumour cells, the sample was considered to have high expression, and if grade 0 or 1 staining was observed in $>50 \%$ of tumour cells, the sample was considered to have low expression (Figure 1). This cutoff value was determined on the basis of a previous report (Santini et al, 2010).

Survival. Disease status was regularly assessed every 3 months by blood tests and computed tomography. If a patient had died, the survival time after surgery and cause of death were recorded. For surviving patients (as of 4 July 2013), postsurgical time and recurrence status were recorded. The failure event for OS was 

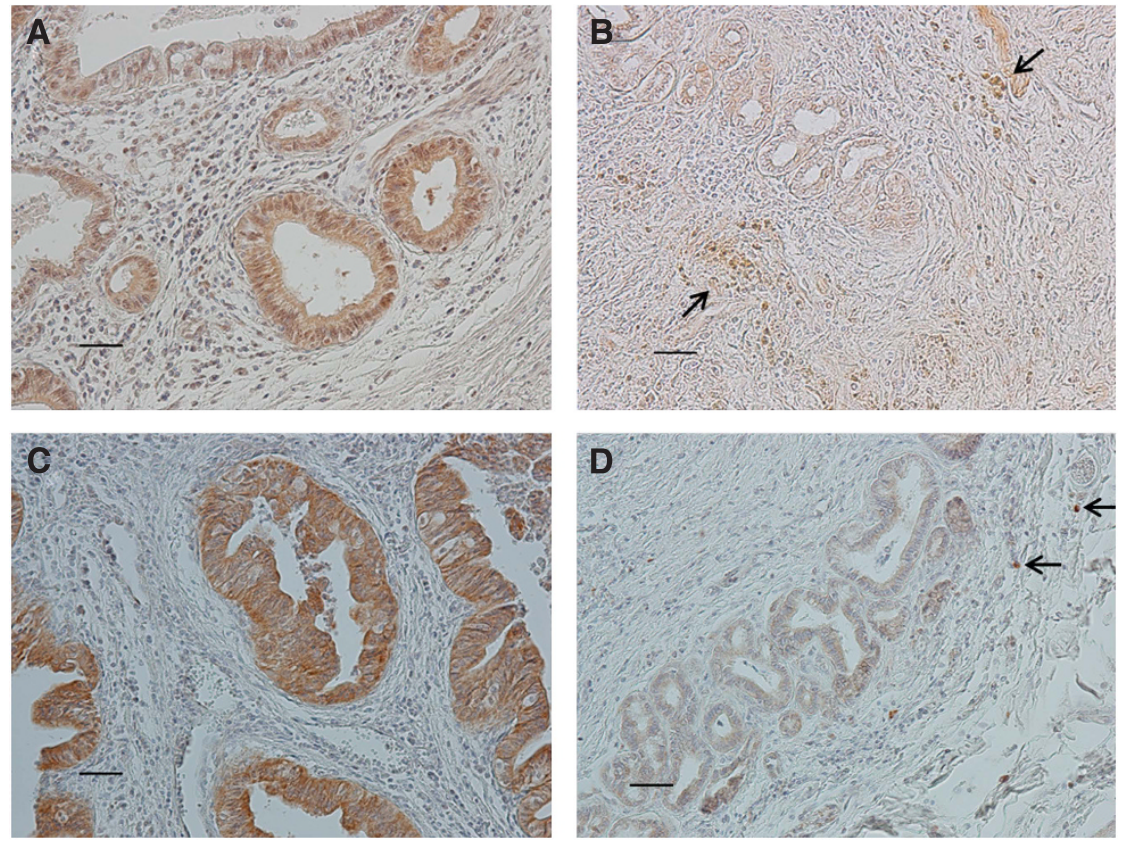

Figure 1. Immunohistochemical analysis of human equilibrative nucleoside transporter 1 (hENT1) and ribonucleotide reductase subunit 1 (RRM1) expression in cholangiocarcinoma. These photomicrographs reveal (A) high hENT1 expression, (B) low hENT1 expression, (C) high RRM1 expression, (D) low RRM1 expression (original magnification, $\times 200$; bar $=50 \mu \mathrm{m}$ ). Positive internal controls is established by staining of lymphocytes and stromal cells (arrows).

defined as death of any cause, whereas that for disease-free survival (DFS) was defined as disease recurrence, diagnosed based on imaging findings, or death of any cause. Survival time was measured from the date of operation to the date of the failure event or last follow-up evaluation.

Statistical analysis. Categorical clinicopathological variables were compared using the $\chi^{2}$-test and Fisher's exact test, as appropriate. Survival end points were estimated using the Kaplan-Meier method and compared by univariate log-rank (Mantel-Cox) test. The Cox proportional hazards model was applied to the multivariate survival analysis for factors found to be significant on univariate analysis. The UICC stage was excluded in the multivariate analysis, although it was significant by univariate analysis, because of its confounding with UICC pT factor and lymph node metastasis. $P$-values of $<0.05$ were considered statistically significant. Statistical analyses were performed by JMP software, version 10.2 (SAS Institute, Cary, NC, USA).

\section{RESULTS}

Patient demographics and pathological assessment. A total of 132 consecutive patients with UICC stages II-IV cholangiocarcinoma underwent surgical resection (R0 or R1 resection) at our institution between April 1989 and August 2012. Of these 132 patients, $5(3.7 \%)$ were excluded from this study because of operative deaths. In total, 127 cholangiocarcinoma patients were eligible for this study. This case series included 105 (83\%) patients previously reported in our retrospective analysis of hENT1 expression in cholangiocarcinoma (Kobayashi et al, 2012). Demographics and clinicopathological factors of enrolled patients are summarised in Table 1 . The median age of these 127 patients was 69 years (range: 37-85 years). Lymph node metastasis was found in $65(51 \%)$ patients, including $12(9 \%)$ with para-aortic lymph node involvement. Finally, 32 (25\%), 17 (13\%), 24 (19\%), 2 (2\%), 7 (6\%), $20(16 \%), 6(5 \%), 13(10 \%)$, and $6(5 \%)$ patients were diagnosed with stage II, IIA, IIB, III, IIIA, IIIB, IV, IVA, and IVB disease, respectively. All 12 patients with stage IV or IVB disease had para-aortic lymph node metastases detectable only by postoperative histological examination, but not by pre-operativeimaging examinations.

Delivery of AGC. Of the 127 patients, 68 (54\%) received postoperative AGC, and 59 (46\%) did not. In the 68 patients who received AGC, 60 (88\%) patients received adjuvant gemcitabine plus S-1 chemotherapy, and 8 (12\%) patients received gemcitabine alone. Sixty-one (90\%) patients received 10 cycles of AGC, whereas the remaining $7(10 \%)$ patients had to switch to other chemotherapies at 7 or 8 cycles of AGC because of recurrent disease. The median total dose of gemcitabine administered to the 68 patients was $17000 \mathrm{mg}$ (range: 7000-44 $000 \mathrm{mg}$ ). No treatmentrelated deaths were reported in this case series. In the 59 patients without AGC, 55 (93\%) patients received only surgical treatment, and $4(7 \%)$ patients received adjuvant oral UFT chemotherapy.

Clinicopathological factors and intratumoural hENT1 and RRM1 expression. Potential correlations of hENT1 and RRM1 expression levels with each clinicopathological factor are shown in Table 1. High intratumoural hENT1 and RRM1 expression levels were observed in $86(68 \%)$ and 67 (53\%) patients, respectively. Significant differences in hENT1 expression were found among samples with varying states of pathological differentiation $(P=0.009)$, and poorly differentiated adenocarcinoma samples were more likely to exhibit low RRM1 expression $(P=0.017)$. In addition, the distribution of RRM1 expression was significantly different depending on the tumour location $(P=0.020)$, pathological differentiation $(P=0.017)$, and UICC stage $(P=0.009)$. Other clinicopathological factors did not correlate with hENT1 or RRM1 expression.

Univariate survival analysis for patients with or without AGC. The median follow-up time after surgery was 81 months (range: 9-294 months) for all 127 patients. The 5-year DFS and OS rates for these patients were $26 \%$ and $33 \%$, respectively. The results of univariate DFS and OS analyses for patients with or without AGC are shown in Table 2. In 68 patients who received AGC, 
Table 1. Comparison of clinicopathological factors based on intratumoural hENT1 and RRM1 expression for all patients $(n=127)$

No. of patients (\%)

No. of patients (\%)

\begin{tabular}{|l|c|c|c|c|c|c|c|}
\hline & & High hENT1 & Low hENT1 & & High RRM1 & Low RRM1 & \\
\hline & Total no. of patients (\%) & $n=86(68)$ & $n=41(32)$ & $P$-value & $n=67(53)$ & $n=60(47)$ & $P$-value \\
\hline
\end{tabular}

Ages(years)

\begin{tabular}{|c|c|c|c|c|c|c|c|}
\hline$<70$ & $66(52)$ & $48(56)$ & $18(44)$ & 0.209 & $38(57)$ & $28(47)$ & 0.258 \\
\hline$\geqslant 70$ & $61(48)$ & 38 (44) & $23(56)$ & & $29(43)$ & $32(53)$ & \\
\hline
\end{tabular}

Gender

\begin{tabular}{|l|l|l|l|l|l|l|l|l|l|}
\hline Male & $85(67)$ & $56(65)$ & $29(71)$ & 0.529 & $43(64)$ \\
Female & $42(33)$ & $30(35)$ & $12(29)$ & 0.486 \\
\hline
\end{tabular}

Tumour location

\begin{tabular}{l|l|l|l|l|l|r|}
\hline Intrahepatic & $20(16)$ & $13(15)$ & $7(17)$ & 0.251 & $14(21)$ \\
Perihilar & $60(47)$ & $37(43)$ & $23(56)$ & $24(36)$ & $36(60)$ \\
Distal & $47(37)$ & $36(42)$ & $11(27)$ & $18(30)$ \\
\hline
\end{tabular}

AGC
\begin{tabular}{|l|l|l|l|l|l|l|l|}
\hline Yes & $68(54)$ & $45(52)$ & $23(56)$ & 0.69 & $35(52)$ & $33(55)$ & 0.755 \\
No & $59(46)$ & $41(48)$ & $18(44)$ & & $32(48)$ & $27(45)$ & \\
\hline
\end{tabular}

Residual tumour

\begin{tabular}{|l|l|l|l|l|l|l|l|}
\hline R0 & $95(75)$ & $64(74)$ & $31(76)$ & 0.885 & $54(81)$ & $41(68)$ & 0.112 \\
R1 & $32(25)$ & $22(26)$ & $10(24)$ & & $13(19)$ & $19(32)$ \\
\hline
\end{tabular}

Pathological differentiation

\begin{tabular}{|c|c|c|c|c|c|c|c|}
\hline $\begin{array}{l}\text { Well } \\
\text { Moderately } \\
\text { Poorly }\end{array}$ & $\begin{array}{l}55(43) \\
49(39) \\
23(18)\end{array}$ & $\begin{array}{l}45(52) \\
29(34) \\
12(14)\end{array}$ & $\begin{array}{l}10(24) \\
20(49) \\
11(27)\end{array}$ & 0.009 & $\begin{array}{c}33(49) \\
28(42) \\
6(9)\end{array}$ & $\begin{array}{l}22 \text { (37) } \\
21(35) \\
17(28)\end{array}$ & 0.017 \\
\hline \multicolumn{8}{|c|}{ Lymph node metastasis } \\
\hline $\begin{array}{l}\text { Present } \\
\text { Absent }\end{array}$ & $\begin{array}{l}65(51) \\
62(49)\end{array}$ & $\begin{array}{l}47(55) \\
39(45)\end{array}$ & $\begin{array}{l}18(44) \\
23(56)\end{array}$ & 0.257 & $\begin{array}{l}36(54) \\
31(46)\end{array}$ & $\begin{array}{ll}29 & (48) \\
31 & (52)\end{array}$ & 0.544 \\
\hline
\end{tabular}

\section{UICC $p$ T factor}

\begin{tabular}{|c|c|c|c|c|c|c|c|}
\hline $\begin{array}{l}\text { T1 } \\
\text { T2, 2a, 2b } \\
\text { T3 } \\
\text { T4 }\end{array}$ & $\begin{array}{c}3(2) \\
62(49) \\
59(46) \\
3(2)\end{array}$ & $\begin{array}{c}2(2) \\
38(44) \\
43(50) \\
3(4)\end{array}$ & $\begin{array}{c}1(2) \\
24(59) \\
16(39) \\
0(0)\end{array}$ & 0.345 & $\begin{array}{c}3(4) \\
32(48) \\
29(43) \\
3(4)\end{array}$ & $\begin{array}{c}0(0) \\
30(50) \\
30(50) \\
0(0)\end{array}$ & 0.126 \\
\hline \multicolumn{8}{|c|}{ UICC stages } \\
\hline $\begin{array}{l}\text { II, IIA, IIB } \\
\text { III, IIIA, IIIB } \\
\text { IV, IVA, IVB }\end{array}$ & $\begin{array}{l}73(57) \\
29(23) \\
25(20)\end{array}$ & $\begin{array}{l}49(57) \\
18(21) \\
19(22)\end{array}$ & $\begin{array}{r}24(58) \\
11(27) \\
6(15) \\
\end{array}$ & 0.544 & $\begin{array}{r}40(60) \\
9(13) \\
18(27) \\
\end{array}$ & $\begin{array}{r}33(55) \\
20(33) \\
7(12) \\
\end{array}$ & 0.009 \\
\hline
\end{tabular}

Abbreviations: AGC = adjuvant gemcitabine-based chemotherapy; hENT1 = human equilibrative nucleoside transporter 1; RRM1=ribonucleotide reductase subunit 1.

pathological differentiation $(P=0.003)$, UICC stage $(P=0.042)$, hENT1 expression $(P=0.005)$, and RRM1 expression $(P=0.015)$ were significantly associated with DFS, and pathological differentiation $(P=0.011)$, lymph node metastasis $(P=0.009)$, UICC stage $(P=0.012)$, hENT1 expression $(P=0.036)$, and RRM1 expression $(P=0.035)$ were also significantly associated with OS. In the 59 patients who did not receive AGC, residual tumour $(P<0.001)$ and lymph node metastasis $(P=0.037)$ were significantly associated with DFS, and residual tumour $(P<0.001)$, pathological differentiation $(P=0.049)$, lymph node metastasis $(P=0.007)$, and UICC stage $(P=0.017)$ were significantly associated with OS. However, both hENT1 and RRM1 expression were not significantly correlated with DFS (hENT1: $P=0.796$,
RRM1: $P=0.642$ ) or OS (hENT1: $P=0.913$, RRM1: $P=0.883$ ) (Figures 2 and 3 ).

Each of the 68 patients who received AGC was classified into four groups based on hENT1 and RRM1 expression levels as follows: high hENT1/low RRM1 expression $(n=20)$, high hENT1/ high RRM1 expression ( $n=25)$, low hENT1/low RRM1 expression $(n=13)$, and low hENT1/high RRM1 expression $(n=10)$, which was significantly associated with both DFS $(P=0.003)$ and OS $(P=0.015)$ by univariate analysis (Figure 4$)$. Moreover, patients with high hENT1/low RRM1 expression experienced significantly longer DFS and OS than those with high hENT1/high RRM1 expression (DFS: $P=0.001$, OS: $P=0.006$ ), low hENT1/low RRM1 expression (DFS: $P<0.001$, OS: $P=0.002$ ), and low hENT1/high 
Table 2. Univariate DFS and OS analysis of prognostic factors in patients with cholangiocarcinoma who received AGC $(n=68)$ and those who did not $(n=59)$

\begin{tabular}{|c|c|c|c|c|c|c|c|c|c|c|}
\hline & \multicolumn{5}{|c|}{$\operatorname{AGC}(+)(n=68)$} & \multicolumn{5}{|c|}{$\operatorname{AGC}(-)(n=59)$} \\
\hline & & \multicolumn{2}{|c|}{ DFS } & \multicolumn{2}{|c|}{ OS } & \multicolumn{3}{|c|}{ DFS } & \multicolumn{2}{|c|}{ os } \\
\hline & $N$ & $\begin{array}{l}\text { 5-Year } \\
\text { survival } \\
(\%)\end{array}$ & $P$-value & $\begin{array}{l}\text { 5-Year } \\
\text { survival } \\
(\%)\end{array}$ & $P$-value & $N$ & $\begin{array}{l}\text { 5-Year } \\
\text { survival } \\
(\%)\end{array}$ & $P$-value & $\begin{array}{l}\text { 5-Year } \\
\text { survival } \\
(\%)\end{array}$ & $P$-value \\
\hline \multicolumn{11}{|l|}{ Age (years) } \\
\hline $\begin{array}{l}<70 \\
\geqslant 70\end{array}$ & $\begin{array}{l}35 \\
33\end{array}$ & $\begin{array}{l}38 \\
36\end{array}$ & 0.941 & $\begin{array}{l}61 \\
30\end{array}$ & 0.225 & $\begin{array}{l}31 \\
28\end{array}$ & $\begin{array}{l}18 \\
14\end{array}$ & 0.834 & $\begin{array}{l}26 \\
15\end{array}$ & 0.472 \\
\hline \multicolumn{11}{|l|}{ Gender } \\
\hline $\begin{array}{l}\text { Male } \\
\text { Female }\end{array}$ & $\begin{array}{l}42 \\
26\end{array}$ & $\begin{array}{l}40 \\
19\end{array}$ & 0.518 & $\begin{array}{l}53 \\
35\end{array}$ & 0.566 & $\begin{array}{l}43 \\
16\end{array}$ & $\begin{array}{l}17 \\
13\end{array}$ & 0.483 & $\begin{array}{l}20 \\
21\end{array}$ & 0.56 \\
\hline \multicolumn{11}{|l|}{ Tumour location } \\
\hline $\begin{array}{l}\text { Intrahepatic } \\
\text { Perihilar } \\
\text { Distal }\end{array}$ & $\begin{array}{l}12 \\
36 \\
20\end{array}$ & $\begin{array}{l}20 \\
43 \\
33\end{array}$ & 0.129 & $\begin{array}{l}56 \\
49 \\
39\end{array}$ & 0.479 & $\begin{array}{r}8 \\
24 \\
27\end{array}$ & $\begin{array}{l}25 \\
17 \\
13\end{array}$ & 0.56 & $\begin{array}{l}13 \\
27 \\
16\end{array}$ & 0.841 \\
\hline \multicolumn{11}{|l|}{ Residual tumour } \\
\hline $\begin{array}{l}\text { R0 } \\
\text { R1 }\end{array}$ & $\begin{array}{l}53 \\
15\end{array}$ & $\begin{array}{l}42 \\
16\end{array}$ & 0.077 & $\begin{array}{l}50 \\
36\end{array}$ & 0.11 & $\begin{array}{l}42 \\
17\end{array}$ & $\begin{array}{r}23 \\
0\end{array}$ & 0.001 & $\begin{array}{r}29 \\
0\end{array}$ & $<0.001$ \\
\hline \multicolumn{11}{|l|}{ Pathological differentiation } \\
\hline $\begin{array}{l}\text { Well } \\
\text { Moderate, poor }\end{array}$ & $\begin{array}{l}28 \\
40\end{array}$ & $\begin{array}{l}54 \\
20\end{array}$ & 0.003 & $\begin{array}{l}62 \\
32\end{array}$ & 0.011 & $\begin{array}{l}27 \\
32\end{array}$ & $\begin{array}{r}27 \\
4\end{array}$ & 0.187 & $\begin{array}{r}34 \\
7\end{array}$ & 0.049 \\
\hline \multicolumn{11}{|l|}{ Lymph node metastasis } \\
\hline $\begin{array}{l}\text { Present } \\
\text { Absent }\end{array}$ & $\begin{array}{l}31 \\
37\end{array}$ & $\begin{array}{l}34 \\
40\end{array}$ & 0.057 & $\begin{array}{l}31 \\
60\end{array}$ & 0.009 & $\begin{array}{l}34 \\
25\end{array}$ & $\begin{array}{r}9 \\
26\end{array}$ & 0.037 & $\begin{array}{l}10 \\
35\end{array}$ & 0.007 \\
\hline \multicolumn{11}{|l|}{ UICC pT factor } \\
\hline $\begin{array}{l}\mathrm{T} 1,2,2 \mathrm{a}, 2 \mathrm{~b} \\
\mathrm{~T} 3,4\end{array}$ & $\begin{array}{l}34 \\
34\end{array}$ & $\begin{array}{l}43 \\
28\end{array}$ & 0.216 & $\begin{array}{l}56 \\
35\end{array}$ & 0.126 & $\begin{array}{l}31 \\
28\end{array}$ & $\begin{array}{l}21 \\
10\end{array}$ & 0.092 & $\begin{array}{l}27 \\
12\end{array}$ & 0.167 \\
\hline \multicolumn{11}{|l|}{ UICC stages } \\
\hline $\begin{array}{l}I I, A, B \\
I I I, A, B, I V, A, B\end{array}$ & $\begin{array}{l}35 \\
33\end{array}$ & $\begin{array}{l}43 \\
30\end{array}$ & 0.042 & $\begin{array}{l}66 \\
25\end{array}$ & 0.012 & $\begin{array}{l}38 \\
21\end{array}$ & $\begin{array}{r}21 \\
8\end{array}$ & 0.22 & $\begin{array}{l}27 \\
10\end{array}$ & 0.017 \\
\hline \multicolumn{11}{|l|}{ hENT1 expression } \\
\hline $\begin{array}{l}\text { High } \\
\text { Low }\end{array}$ & $\begin{array}{l}45 \\
23\end{array}$ & $\begin{array}{l}45 \\
21\end{array}$ & 0.005 & $\begin{array}{l}55 \\
32\end{array}$ & 0.036 & $\begin{array}{l}41 \\
18\end{array}$ & $\begin{array}{l}18 \\
12\end{array}$ & 0.796 & $\begin{array}{l}24 \\
12\end{array}$ & 0.913 \\
\hline \multicolumn{11}{|l|}{ RRM1 expression } \\
\hline $\begin{array}{l}\text { High } \\
\text { Low }\end{array}$ & $\begin{array}{l}35 \\
33\end{array}$ & $\begin{array}{l}25 \\
47\end{array}$ & 0.015 & $\begin{array}{l}33 \\
59\end{array}$ & 0.035 & $\begin{array}{l}32 \\
27\end{array}$ & $\begin{array}{l}16 \\
16\end{array}$ & 0.642 & $\begin{array}{l}22 \\
19\end{array}$ & 0.883 \\
\hline \multicolumn{11}{|c|}{ Combined hENT1 and RRM1 classification } \\
\hline $\begin{array}{l}\text { High hENT1/low RRM1 } \\
\text { High hENT1/high RRM1 } \\
\text { Low hENT1/low RRM1 } \\
\text { Low hENT1/high RRM1 }\end{array}$ & $\begin{array}{l}20 \\
25 \\
13 \\
10\end{array}$ & $\begin{array}{l}58 \\
24 \\
19 \\
25\end{array}$ & 0.003 & $\begin{array}{l}75 \\
34 \\
32 \\
36\end{array}$ & 0.015 & $\begin{array}{r}12 \\
29 \\
15 \\
3\end{array}$ & $\begin{array}{r}18 \\
18 \\
13 \\
0\end{array}$ & 0.778 & $\begin{array}{r}25 \\
23 \\
13 \\
0\end{array}$ & 0.994 \\
\hline High hENT1/low RRM1 & 20 & 58 & $<0.001$ & 75 & 0.001 & & & & & \\
\hline The other three expression combinations & 48 & 22 & & 32 & & & & & & \\
\hline
\end{tabular}


A

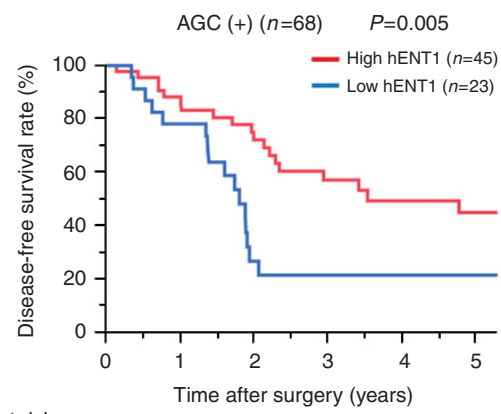

No. at risk

$\begin{array}{lllcccc}\text { High hENT1 } & 45 & 35 & 25 & 17 & 12 & 9 \\ \text { Low hENT1 } & 23 & 17 & 5 & 3 & 2 & 1\end{array}$

C

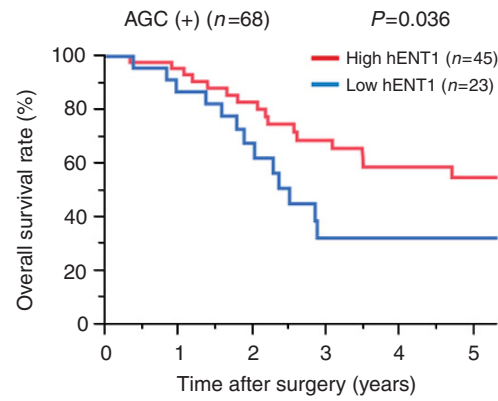

No. at risk

$\begin{array}{lllllll}\text { High hENT1 } & 45 & 41 & 33 & 23 & 15 & 14\end{array}$

$\begin{array}{llllll}\text { Low hENT1 } & 23 & 20 & 12 & 3 & 2\end{array}$

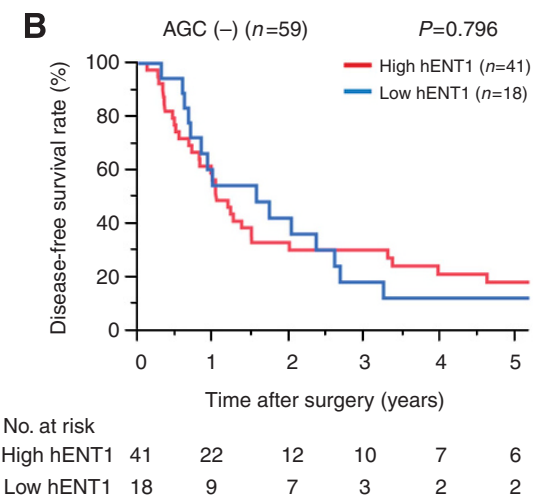

D

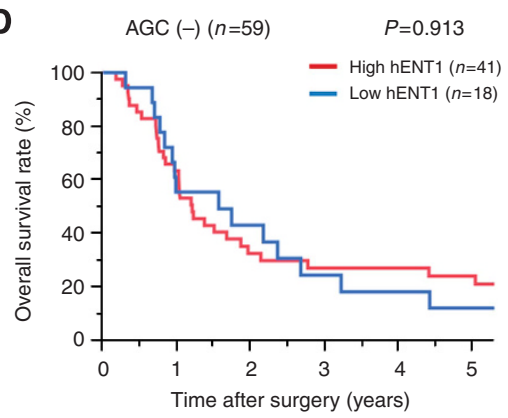

No. at risk

$\begin{array}{lllllll}\text { High hENT1 } & 41 & 25 & 12 & 10 & 9 & 8\end{array}$

$\begin{array}{lllllll}\text { Low hENT1 } & 18 & 10 & 7 & 4 & 3 & 2\end{array}$

Figure 2. Disease-free survival (DFS) and overall survival (OS) curves stratified by intratumoural hENT1 expression. AGC $(+)$ indicates subgroups of patients who received adjuvant gemcitabine-based chemotherapy; AGC $(-)$ indicates subgroups of patients who did not receive adjuvant gemcitabine-based chemotherapy. (A) DFS curves in AGC $(+)$ patients $(P=0.005)$. (B) DFS curves in AGC $(-)$ patients $(P=0.796)$. (C) OS curves in AGC $(+)$ patients $(P=0.036)$. (D) OS curves in AGC $(-)$ patients $(P=0.913)$.

RRM1 expression (DFS: $P<0.001$, OS: $P=0.003$ ). On the basis of these findings, we further categorised these 68 patients who received AGC into the high hENT1/low RRM1 expression $(n=20)$ group and low hENT1 and/or high RRM1 group $(n=48)$ for comparative purposes. This combined classification was significantly associated with both DFS $(P<0.001)$ and OS $(P=0.001)$ by univariate analysis.

On the other hand, each of the 59 patients who did not received AGC was also classified into four groups: high hENT1/low RRM1 expression $(n=12)$, high hENT1/high RRM1 expression $(n=29)$, low hENT1/low RRM1 expression $(n=15)$, and low hENT1/high RRM1 expression $(n=3)$, which was not significantly associated with both DFS $(P=0.778)$ and $\operatorname{OS}(P=0.994)$ by univariate analysis.

Multivariate survival analysis for patients who received AGC. Multivariate analysis including separated hENT1 and RRM1 expression for 68 patients who received AGC identified welldifferentiated (HR, 0.37; 95\% CI, 0.17-0.76; $P=0.007$ ), high hENT1 expression (HR, 0.49; 95\% CI, 0.24-0.98; $P=0.044$ ), and low RRM1 expression (HR, 0.41; 95\% CI, 0.21-0.80; $P=0.009$ ) as independent factors for prolonged DFS and well-differentiated (HR, 0.45; 95\% CI, $0.19-0.98 ; P=0.045$ ), absence of lymph node metastasis (HR, 0.39; 95\% CI, 0.18-0.81; $P=0.011$ ), and low RRM1 expression (HR, 0.43; 95\% CI, $0.20-0.89 ; P=0.024)$ as independent prognostic factors for prolonged OS (Table 3, model 1).

Furthermore, multivariate analysis including combined hENT1 and RRM1 classification was performed among the 68 patients who received AGC. Well-differentiated (HR, 0.38; 95\% CI, 0.17-0.77; $P=0.007)$ and high hENT1/low RRM1 expression (HR, $0.22 ; 95 \%$ CI, $0.08-0.51 ; P<0.001)$ were identified as independent factors of prolonged DFS, and absence of lymph node metastasis (HR, 0.39;
95\% CI, 0.18-0.81; $P=0.012)$ and high hENT1/low RRM1 expression (HR, 0.22; 95\% CI, 0.07-0.60; $P=0.001$ ) were identified as independent factors for prolonged OS (Table 3, model 2).

\section{DISCUSSION}

As some clinical studies, including one randomised controlled trial, have revealed that gemcitabine-based chemotherapy provides a survival advantage for patients with unresectable biliary cancer (Okusaka et al, 2010; Valle et al, 2010), gemcitabine has also been recognised as a key anticancer drug in adjuvant chemotherapy for resectable cholangiocarcinoma. Indeed, our previous studies have revealed that use of AGC was independently associated with prolonged survival (Murakami et al, 2009, 2011, 2012). On the basis of these findings, we believe that AGC can provide a survival benefit for patients with resectable cholangiocarcinoma, and identification of biomarkers that could predict the clinical outcome of AGC may contribute to further optimisation of adjuvant chemotherapy for cholangiocarcinoma. The current study and our previous study (Kobayashi et al, 2012) has revealed the predictive significance of hENT1 in advanced cholangiocarcinoma patients who received AGC in the adjuvant setting. These results suggested that hENT1 expression could be used as a predictive marker for the efficacy of AGC. In contrast to hENT1, however, reports investigating the predictive and prognostic values of intratumoural RRM1 expression with immunochemical staining in biliary cancer are extremely rare. To the best of our knowledge, this is the first clinical report concurrently investigating hENT1 and RRM1 expression in cholangiocarcinoma patients treated with AGC. 
A

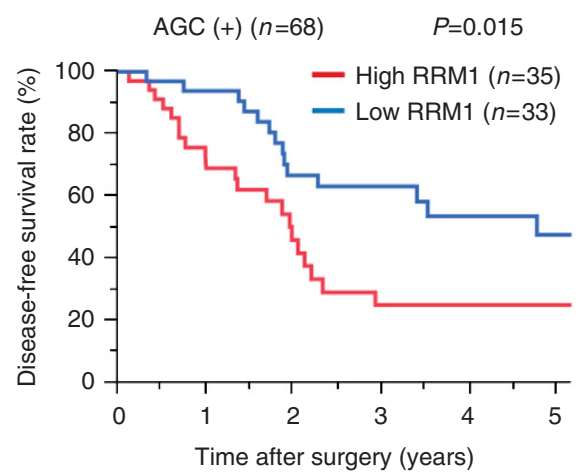

No. at risk

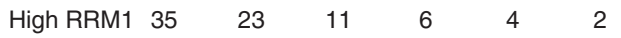

$\begin{array}{lllllll}\text { Low RRM1 } & 33 & 29 & 19 & 14 & 10 & 8\end{array}$

C

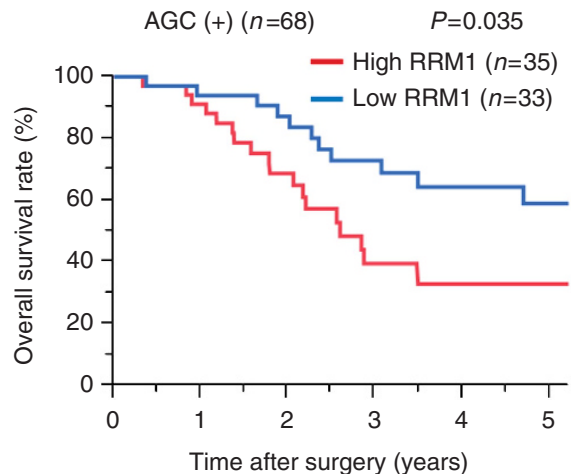

No. at risk

$\begin{array}{llllccc}\text { High RRM1 } & 35 & 30 & 20 & 8 & 5 & 5 \\ \text { Low RRM1 } & 33 & 31 & 25 & 18 & 12 & 11\end{array}$

B

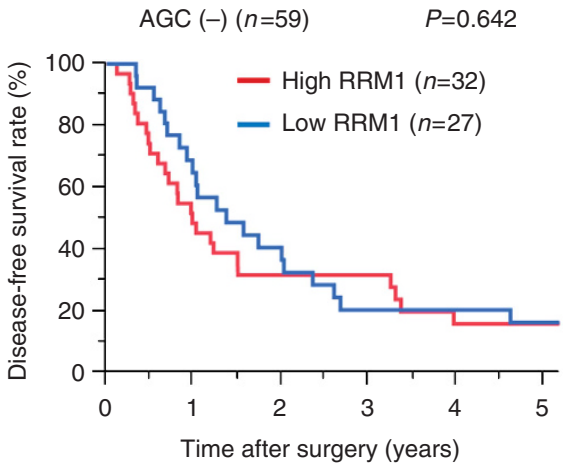

No. at risk

$\begin{array}{llccccc}\text { High RRM1 } & 32 & 15 & 9 & 8 & 4 & 4 \\ \text { Low RRM1 } & 27 & 16 & 10 & 5 & 5 & 4\end{array}$

D

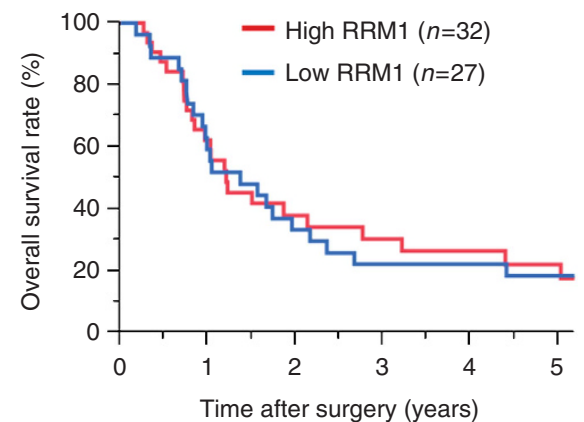

No. at risk

$\begin{array}{llllll}\text { High RRM1 } 32 & 19 & 20 & 8 & 6 & 5\end{array}$

$\begin{array}{lllllll}\text { Low RRM1 } & 29 & 16 & 9 & 6 & 6 & 5\end{array}$

Figure 3. DFS and OS curves stratified by intratumoural RRM1 expression. AGC $(+)$ indicates subgroups of patients who received adjuvant gemcitabine-based chemotherapy; AGC $(-)$ indicates subgroups of patients who did not receive adjuvant gemcitabine-based chemotherapy. (A) DFS curves in AGC $(+)$ patients $(P=0.015)$. (B) DFS curves in AGC $(-)$ patients $(P=0.642)$. (C) OS curves in $A G C(+)$ patients $(P=0.035)$. (D) OS curves in AGC $(-)$ patients $(P=0.883)$.

In this study, $68 \%$ and $53 \%$ of patients with cholangiocarcinoma had high intratumoural hENT1 and RRM1 expression, respectively. One analysis by Fisher et al (2013) of data from 63 patients who underwent surgical resection for biliary malignancies found that $81 \%$ of patients exhibited high RRM1 expression, which was slightly higher than that observed in the current study. Moreover, the current study revealed significant correlations between RRM1 expression and some clinicopathological factors, although no previous reports including a sufficient number of patients have previously demonstrated these correlations. Therefore, further, larger-scale studies on RRM1 expression in cholangiocarcinoma are needed.

RRM1 is the large subunit of human ribonucleotide reductase. In cellular replication, ribonucleotide reductase catalyses the production of deoxynucleotide triphosphates, which are necessary for DNA synthesis. Gemcitabine is currently the most potent and most widely used ribonucleotide reductase inhibitor, and some clinical studies on gastrointestinal and other cancers treated with gemcitabine have demonstrated the significant correlation between increased RRM1 expression and gemcitabine resistance (Ohtaka et al, 2008; Akita et al, 2009; Jordheim et al, 2011; Gong et al, 2012; Nakagawa et al, 2013). However, only a few studies on cholangiocarcinoma, which generally included only a small number of patients, have revealed the predictive significance of RRM1 expression in the palliative setting. The analysis by Ohtaka et al (2008) of data from 12 patients with recurrent biliary carcinoma treated with gemcitabine alone found a trend towards a better response rate in patients with low RRM1 expression compared with those with high RRM1 expression. The analysis by Nakamura et al (2010) of data from 10 patients with advanced biliary carcinoma demonstrated significant associations of low RRM1 expression with gemcitabine sensitivity and improved OS. In contrast, no previous study in the adjuvant setting has evaluated the impact of RRM1 expression on the efficacy and/or prognosis of cholangiocarcinoma patients treated with gemcitabine. The current study revealed that high RRM1 expression was independently associated with poor DFS and OS in patients treated with AGC, but not in those who did not receive AGC. These results suggested that RRM1 expression could be a relevant predictive marker of survival in cholangiocarcinoma patients treated with AGC. On the other hand, some basic studies have demonstrated that increased expression of RRM1 decreases the formation of metastasis and inhibits the development of carcinogen-induced lung tumours (Fan et al, 1997; Gautam et al, 2003; Gautam and Bepler 2006). Indeed, a significant correlation between high RRM1 expression and improved outcomes has been reported in a few studies of lung cancer in patients who underwent surgery alone (Bepler et al, 2004; Zheng et al, 2007). However, no significant difference was 
A

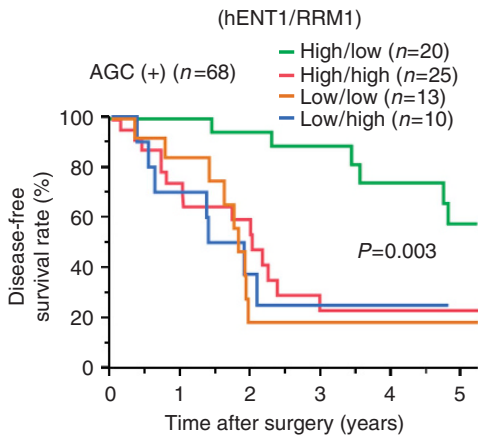

No. at risk (hENT1/RRM1)

$\begin{array}{lcccccc}\text { High/low } & 20 & 19 & 17 & 13 & 9 & 7 \\ \text { High/high } & 25 & 16 & 8 & 4 & 3 & 2 \\ \text { Low/low } & 13 & 10 & 2 & 1 & 1 & 1 \\ \text { Low/high } & 10 & 7 & 3 & 2 & 1 & 0\end{array}$

C

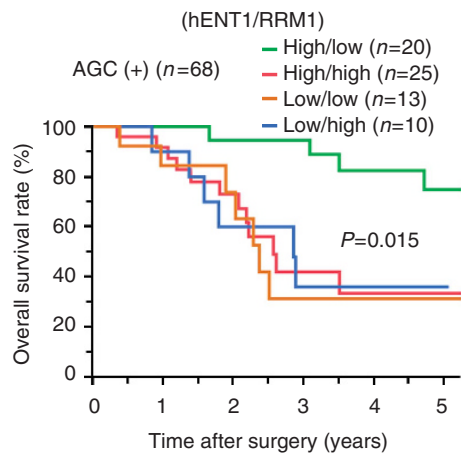

No. at risk (hENT1/RRM1)

$\begin{array}{ccccccc}\text { High/low } & 20 & 20 & 18 & 17 & 11 & 10 \\ \text { High/high } & 25 & 21 & 15 & 6 & 4 & 4 \\ \text { Low/low } & 13 & 11 & 7 & 1 & 1 & 1 \\ \text { Low/high } & 10 & 9 & 5 & 2 & 1 & 1\end{array}$

B

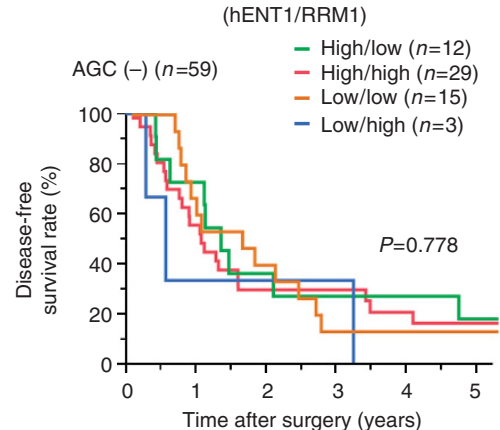

No. at risk

(hENT1/RRM1)

$\begin{array}{lllllll}\text { High/low } & 12 & 18 & 4 & 3 & 3 & 2\end{array}$

$\begin{array}{lllllll}\text { High/high } & 29 & 14 & 8 & 7 & 4 & 4\end{array}$

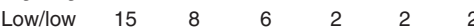

$\begin{array}{lllllll}\text { Low/high } & 3 & 1 & 1 & 1 & 0 & 0\end{array}$

D

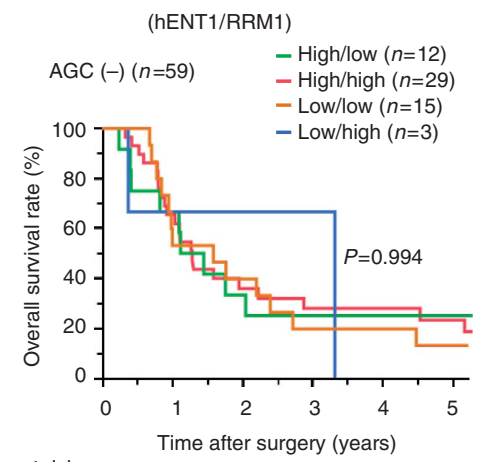

No. at risk (hENT1/RRM1)

$\begin{array}{lcccccc}\text { High/low } & 12 & 8 & 3 & 3 & 3 & 3 \\ \text { High/high } & 29 & 17 & 9 & 7 & 6 & 5 \\ \text { Low/low } & 15 & 8 & 6 & 3 & 3 & 2 \\ \text { Low/high } & 3 & 2 & 1 & 1 & 0 & 0\end{array}$

Figure 4. DFS and OS curves stratified by combined analysis of intratumoural hENT1 and RRM1 expression. AGC $(+)$ indicates subgroups of patients who received adjuvant gemcitabine-based chemotherapy; $A G C(-)$ indicates subgroups of patients who did not receive adjuvant gemcitabine-based chemotherapy. (A) DFS curves in AGC $(+)$ patients $(P=0.003)$. (B) DFS curves in AGC $(-)$ patients $(P=0.778)$. (C) OS curves in AGC $(+)$ patients $(P=0.015)$. (D) OS curves in AGC $(-)$ patients $(P=0.994)$.

found in the current study between RRM1 expression and survival of cholangiocarcinoma patients who did not receive AGC. The possible causes of this discrepancy are differences in cancer type and/or the small number of patients in this study. In addition, patients who did not receive AGC were selected without randomisation and their post-operative courses were slightly different (a few patients received adjuvant oral UFT chemotherapy). Therefore, further basic and clinical studies on the role of RRM1 in the growth and proliferation of cholangiocarcinoma cells are needed.

As both hENT1 and RRM1 were associated with survival in patients treated with AGC, the combined hENT1 and RRM1 classification was introduced in this study to reinforce the predictive values of these targets. The current results demonstrated that patients with high hENT1 and low RRM1 experienced longer DFS and OS compared with the other three groups. In addition, these patients had markedly reduced HRs compared with analyses of separated hENT1 and RRM1 expression. On the basis of these findings, the combined hENT1 and RRM1 classification enabled us to increase the predictive value of these targets for prognosis in cholangiocarcinoma patients treated with AGC compared with either factor alone and may contribute to the optimisation of adjuvant chemotherapy for resected cholangiocarcinoma.
This study has some inherent limitations owing to the small number of patients analysed and the study's retrospective nature. First, patients who did not receive AGC (the control group in the current study) were selected without randomisation. Second, oral fluoropyrimidines were administered to some of the patients treated with and without gemcitabine in the current study. However, we believe this supplement had no effect on the results of this study, because prior studies have revealed that hENT1 and RRM1 work as a predictive marker of gemcitabine but not fluoropyrimidine (Farrell et al, 2009; Fujita et al, 2010; Greenhalf et al, 2014). Third, some other biomarkers, including deoxycytidine kinase, 5'-nucleotidase, cytidine deaminase, and ribonucleotide reductases subunit 2 , have been reported to be associated with response to gemcitabine in pancreatic cancer (Giovannetti et al, 2006; Fujita et al, 2010; Maréchal et al, 2012). The role of these candidates as potential predictive markers in cholangiocarcinoma is still unclear. Further prospective, large-scale, randomised studies are needed to overcome these limitations.

In conclusion, both intratumoural hENT1 and RRM1 expression levels were closely associated with the survival of patients with advanced cholangiocarcinoma treated with AGC after surgical resection. In addition, combined analysis of hENT1 and RRM1 expression was more useful for prediction of AGC efficacy than either factor alone. These findings warrant further 
Table 3. Multivariate DFS and OS analysis of prognostic factors in patients who received AGC $(n=68)$

\begin{tabular}{|c|c|c|c|c|c|c|}
\hline & \multicolumn{3}{|c|}{ DFS } & \multicolumn{3}{|c|}{ OS } \\
\hline & HR & $95 \% \mathrm{Cl}$ & $P$-value & HR & $95 \% \mathrm{Cl}$ & $P$-value \\
\hline \multicolumn{7}{|c|}{ Model 1-multivariate analysis including separated hENT1 and RRM1 expression } \\
\hline \multicolumn{7}{|l|}{ Pathological differentiation } \\
\hline $\begin{array}{l}\text { Well } \\
\text { Moderate, poor }\end{array}$ & $\begin{array}{c}0.37 \\
1\end{array}$ & $0.17-0.76$ & 0.007 & $\begin{array}{c}0.45 \\
1\end{array}$ & $0.19-0.98$ & 0.045 \\
\hline \multicolumn{7}{|l|}{ Lymph node metastasis } \\
\hline $\begin{array}{l}\text { Present } \\
\text { Absent }\end{array}$ & & & & $\begin{array}{c}1 \\
0.39\end{array}$ & $0.18-0.81$ & 0.011 \\
\hline \multicolumn{7}{|l|}{ hENT1 expression } \\
\hline $\begin{array}{l}\text { High } \\
\text { Low }\end{array}$ & $\begin{array}{c}0.49 \\
1\end{array}$ & $0.24-0.98$ & 0.044 & $\begin{array}{c}0.55 \\
1\end{array}$ & $0.26-1.21$ & 0.135 \\
\hline \multicolumn{7}{|l|}{ RRM1 expression } \\
\hline $\begin{array}{l}\text { High } \\
\text { Low }\end{array}$ & $\begin{array}{c}1 \\
0.41\end{array}$ & $0.21-080$ & 0.009 & $\begin{array}{c}1 \\
0.43\end{array}$ & $0.20-0.89$ & 0.024 \\
\hline \multicolumn{7}{|c|}{ Model 2-multivariate analysis including combined hENT1 and RRM1 classification } \\
\hline \multicolumn{7}{|l|}{ Pathological differentiation } \\
\hline $\begin{array}{l}\text { Well } \\
\text { Moderate, poor }\end{array}$ & $\begin{array}{c}0.38 \\
1\end{array}$ & $0.17-0.77$ & 0.007 & $\begin{array}{c}0.49 \\
1\end{array}$ & $0.21-1.05$ & 0.066 \\
\hline \multicolumn{7}{|l|}{ Lymph node metastasis } \\
\hline $\begin{array}{l}\text { Present } \\
\text { Absent }\end{array}$ & & & & $\begin{array}{c}1 \\
0.39\end{array}$ & $0.18-0.81$ & 0.012 \\
\hline \multicolumn{7}{|l|}{ Combined hENT1 and RRM1 classification } \\
\hline $\begin{array}{l}\text { High hENT1/low RRM1 } \\
\text { The other three expression combinations }\end{array}$ & $\begin{array}{c}0.22 \\
1\end{array}$ & $0.08-0.51$ & $<0.001$ & $\begin{array}{c}0.22 \\
1\end{array}$ & $0.07-0.60$ & 0.001 \\
\hline
\end{tabular}

investigations to establish appropriate post-operative treatments for resectable cholangiocarcinoma, which can be optimised based on hENT1 and RRM1 expression levels.

\section{CONFLICT OF INTEREST}

The authors declare no conflict of interest.

\section{REFERENCES}

Akita H, Zheng Z, Takeda Y, Kim C, Kittaka N, Kobayashi S, Marubashi S, Takemasa I, Nagano H, Dono K, Nakamori S, Monden M, Mori M, Doki Y, Bepler G (2009) Significance of RRM1 and ERCC1 expression in resectable pancreatic adenocarcinoma. Oncogene 28: 2903-2909.

Bepler G, Sharma S, Cantor A, Gautam A, Haura E, Simon G, Sharma A, Sommers E, Robinson L (2004) RRM1 and PTEN as prognostic parameters for overall and disease-free survival in patients with non-small-cell lung cancer. J Clin Oncol 22: 1878-1885.

DeOliveira ML, Cunningham SC, Cameron JL, Kamangar F, Winter JM, Lillemoe KD, Choti MA, Yeo CJ, Schulick RD (2007)

Cholangiocarcinoma: thirty-one-year experience with 564 patients at a single institution. Ann Surg 245: 755-762.

Fan H, Huang A, Villegas C, Wright JA (1997) The R1 component of mammalian ribonucleotide reductase has malignancy-suppressing activity as demonstrated by gene transfer experiments. Proc Natl Acad Sci USA 94: 13181-13186.
Farrell JJ, Elsaleh H, Garcia M, Lai R, Ammar A, Regine WF, Abrams R, Benson AB, Macdonald J, Cass CE, Dicker AP, Mackey JR (2009) Human equilibrative nucleoside transporter 1 levels predict response to gemcitabine in patients with pancreatic cancer. Gastroenterology 136: 187-195.

Fisher SB, Fisher KE, Patel SH, Lim MG, Kooby DA, El-Rayes BF, Staley 3rd CA, Adsay NV, Farris 3rd AB, Maithel SK (2013) Excision repair cross-complementing gene-1, ribonucleotide reductase subunit M1, ribonucleotide reductase subunit $\mathrm{M} 2$, and human equilibrative nucleoside transporter-1 expression and prognostic value in biliary tract malignancy. Cancer 119: 454-462.

Fujita H, Ohuchida K, Mizumoto K, Itaba S, Ito T, Nakata K, Yu J, Kayashima T, Souzaki R, Tajiri T, Manabe T, Ohtsuka T, Tanaka M (2010) Gene expression levels as predictive markers of outcome in pancreatic cancer after gemcitabine-based adjuvant chemotherapy. Neoplasia 12: 807-817.

Gautam A, Bepler G (2006) Suppression of lung tumor formation by the regulatory subunit of ribonucleotide reductase. Cancer Res 66: 6497-6502.

Gautam A, Li ZR, Bepler G (2003) RRM1-induced metastasis suppression through PTEN-regulated pathways. Oncogene 22: 2135-2142.

Giovannetti E, Del Tacca M, Mey V, Funel N, Nannizzi S, Ricci S, Orlandini C, Boggi U, Campani D, Del Chiaro M, Iannopollo M, Bevilacqua G, Mosca F, Danesi R (2006) Transcription analysis of human equilibrative nucleoside transporter-1 predicts survival in pancreas cancer patients treated with gemcitabine. Cancer Res 66: 3928-3935.

Gong W, Zhang X, Wu J, Chen L, Li L, Sun J, Lv Y, Wei X, Du Y, Jin H, Dong J (2012) RRM1 expression and clinical outcome of gemcitabine-containing chemotherapy for advanced non-small-cell lung cancer: a meta-analysis. Lung Cancer 75: 374-380.

Greenhalf W, Ghaneh P, Neoptolemos JP, Palmer DH, Cox TF, Lamb RF, Garner E, Campbell F, Mackey JR, Costello E, Moore MJ, Valle JW, 
McDonald AC, Carter R, Tebbutt NC, Goldstein D, Shannon J, Dervenis C, Glimelius B, Deakin M, Charnley RM, Lacaine F, Scarfe AG, Middleton MR, Anthoney A, Halloran CM, Mayerle J, Oláh A, Jackson R, Rawcliffe CL, Scarpa A, Bassi C, Büchler MW. European Study Group for Pancreatic Cancer (2014) Pancreatic cancer hENT1 expression and survival from gemcitabine in patients from the ESPAC-3 trial. J Natl Cancer Inst 106: djt347.

Jordheim LP, Sève P, Trédan O, Dumontet C (2011) The ribonucleotide reductase large subunit (RRM1) as a predictive factor in patients with cancer. Lancet Oncol 12: 693-702.

Kobayashi H, Murakami Y, Uemura K, Sudo T, Hashimoto Y, Kondo N, Sueda T (2012) Human equilibrative nucleoside transporter 1 expression predicts survival of advanced cholangiocarcinoma patients treated with gemcitabine-based adjuvant chemotherapy after surgical resection. Ann Surg 256: 288-296.

Maréchal R, Bachet JB, Mackey JR, Dalban C, Demetter P, Graham K, Couvelard A, Svrcek M, Bardier-Dupas A, Hammel P, Sauvanet A, Louvet C, Paye F, Rougier P, Penna C, André T, Dumontet C, Cass CE, Jordheim LP, Matera EL, Closset J, Salmon I, Devière J, Emile JF, Van Laethem JL (2012) Levels of gemcitabine transport and metabolism proteins predict survival times of patients treated with gemcitabine for pancreatic adenocarcinoma. Gastroenterology 143: 664-674.

Murakami Y, Uemura K, Sudo T, Hashimoto Y, Nakashima A, Kondo N, Sakabe R, Ohge H, Sueda T (2011) Prognostic factors after surgical resection for intrahepatic, hilar, and distal cholangiocarcinoma. Ann Surg Oncol 18: 651-658.

Murakami Y, Uemura K, Sudo T, Hashimoto Y, Nakashima A, Sakabe R, Kobayashi H, Kondo N, Nakagawa N, Sueda T (2012) Adjuvant chemotherapy with gemcitabine and S-1 after surgical resection for advanced biliary carcinoma: outcomes and prognostic factors. J Hepatobiliary Pancreat Sci 19: 306-313.

Murakami Y, Uemura K, Sudo T, Hayashidani Y, Hashimoto Y, Nakamura H, Nakashima A, Sueda T (2009) Adjuvant gemcitabine plus S-1 chemotherapy improves survival after aggressive surgical resection for advanced biliary carcinoma. Ann Surg 250: 950-956.

Nagino M, Ebata T, Yokoyama Y, Igami T, Sugawara G, Takahashi Y, Nimura Y (2013) Evolution of surgical treatment for perihilar cholangiocarcinoma: a single-center 34-year review of 574 consecutive resections. Ann Surg 258: 129-140.

Nakagawa N, Murakami Y, Uemura K, Sudo T, Hashimoto Y, Kondo N, Sueda T (2013) Combined analysis of intratumoral human equilibrative nucleoside transporter 1 (hENT1) and ribonucleotide reductase regulatory subunit M1 (RRM1) expression is a powerful predictor of survival in patients with pancreatic carcinoma treated with adjuvant gemcitabinebased chemotherapy after operative resection. Surgery 153: 565-575.

Nakamura J, Kohya N, Kai K, Ohtaka K, Hashiguchi K, Hiraki M, Kitajima Y, Tokunaga O, Noshiro H, Miyazaki K (2010) Ribonucleotide reductase subunit M1 assessed by quantitative double-fluorescence immunohistochemistry predicts the efficacy of gemcitabine in biliary tract carcinoma. Int J Oncol 37: 845-852.

National Cancer Center, Japan (2014) Cancer Mortality: Center for Cancer Control and Information Services. Available at http://ganjoho.jp/ professional/statistics/statistics.htmlaccessed on 9 May 2014) (Japanese).

Ohtaka K, Kohya N, Sato K, Kitajima Y, Ide T, Mitsuno M, Miyazaki K (2008) Ribonucleotide reductase subunit M1 is a possible chemoresistance marker to gemcitabine in biliary tract carcinoma. Oncol Rep 20: 279-286.

Okusaka T, Nakachi K, Fukutomi A, Mizuno N, Ohkawa S, Funakoshi A, Nagino M, Kondo S, Nagaoka S, Funai J, Koshiji M, Nambu Y, Furuse J, Miyazaki M, Nimura Y (2010) Gemcitabine alone or in combination with cisplatin in patients with biliary tract cancer: a comparative multicentre study in Japan. Br J Cancer 103: 469-474.

Santini D, Vincenzi B, Fratto ME, Perrone G, Lai R, Catalano V, Cass C, Ruffini PA, Spoto C, Muretto P, Rizzo S, Muda AO, Mackey JR, Russo A, Tonini G, Graziano F (2010) Prognostic role of human equilibrative nucleoside transporter 1 (hENT1) in patients with resected gastric cancer. J Cell Physiol 223: 384-388.

Siegel R, Naishadham D, Jemal A (2013) Cancer statistics, 2013. CA Cancer J Clin 63: 11-30.

Sobin LH, Gospodarowicz MK, Wittekind C (eds) (2010) International Union Against Cancer (UICC) TNM Classification of Malignant Tumors.. 7th edn. Wiley-Blackwell: New York, NY, USA.

Spratlin J, Sangha R, Glubrecht D, Dabbagh L, Young JD, Dumontet C, Cass C, Lai R, Mackey JR (2004) The absence of human equilibrative nucleoside transporter 1 is associated with reduced survival in patients with gemcitabine-treated pancreas adenocarcinoma. Clin Cancer Res 10: 6956-6961.

Valle J, Wasan H, Palmer DH, Cunningham D, Anthoney A, Maraveyas A, Madhusudan S, Iveson T, Hughes S, Pereira SP, Roughton M, Bridgewater J. ABC-02 Trial Investigators (2010) Cisplatin plus gemcitabine versus gemcitabine for biliary tract cancer. N Engl J Med 362: 1273-1281.

Wei CH, Gorgan TR, Elashoff DA, Hines OJ, Farrell JJ, Donahue TR (2013) A meta-analysis of gemcitabine biomarkers in patients with pancreaticobiliary cancers. Pancreas 42: 1303-1310.

Zheng Z, Chen T, Li X, Haura E, Sharma A, Bepler G (2007) DNA synthesis and repair genes RRM1 and ERCC1 in lung cancer. N Engl J Med 356: 800-808.

This work is published under the standard license to publish agreement. After 12 months the work will become freely available and the license terms will switch to a Creative Commons AttributionNonCommercial-Share Alike 3.0 Unported License. 